\title{
Comportamientos de riesgo para la salud en los adolescentes
}

\author{
Health risk behaviors in adolescents \\ María Cecilia Paredes-Iragorri1 orcid.org/0000-0002-0277-0469 \\ Luz Adriana Patiño-Guerrero'** orcid.org/0000-0002-0939-1461
}

1 Departamento de Pediatría, Facultad de Medicina, Universidad de La Sabana. Chía Colombia.

Fecha de recepción: Enero 23 - $2019 \quad$ Fecha de revisión: Mayo 5 - $2019 \quad$ Fecha de aceptación: Diciembre 19 - 2019

Paredes-Iragorri MC, Patiño-Guerrero LA. Comportamientos de riesgo para la salud en los adolescentes. Univ. Salud. 2020;22(1):58-69. DOI: https://doi.org/10.22267/rus.202201.175

\section{Resumen}

Introducción: Los adolescentes experimentan cambios físicos y psicosociales que los hacen vulnerables a múltiples comportamientos de riesgo, evidenciando la necesidad de generar políticas de prevención. Objetivo: Identificar los principales comportamientos de riesgo de los adolescentes, su prevalencia, factores predisponentes; así como las guías y escalas validadas que existen para su detección. Materiales y métodos: Se realizó una búsqueda bibliográfica en diferentes bases de datos y páginas oficiales de las principales organizaciones de salud. Se seleccionaron 45 artículos completos en español e inglés, publicados entre los años 2003 y 2017. Resultados: Se agruparon los comportamientos de riesgo en cinco dominios de los cuales se identificó su prevalencia se describieron las guías y escalas validadas existentes para su detección. Conclusiones: Al identificar los comportamientos de riesgo en los adolescentes se disminuye su morbimortalidad si se intervienen de forma precoz. Se recomienda a los profesionales de salud utilizar las mejores herramientas de tamizaje disponibles, que evalúan de manera rápida y simultánea múltiples comportamientos tales como las guías: Education/employment, peer Activities, Drugs, Sexuality and Suicide/depression, Rapid Assessment for Adolescent Preventive Services, Global school-based student health survey, de fácil aplicación en atención primaria y permiten realizar un adecuado abordaje y manejo de esta población.

Palabras clave: Adolescente; comportamiento del adolescente; conductas de riesgo para la salud; prevalencia; factores protectores; evaluación de riesgo. (Fuente: DeCS, Bireme).

\begin{abstract}
Introduction: Adolescents experience physical and psychosocial changes that make them vulnerable to multiple risk behaviors, highlighting the need to generate prevention policies. Objective: To identify main risk behaviors of adolescents and their prevalence, as well as the validated guides and scales for their detection. Materials and methods: A bibliographic search was carried out in different databases and official pages of main health organizations. 45 complete articles published in Spanish and English from 2013 to 2017 were selected. Results: Risk behaviors were grouped into five domains, identifying their prevalence and describing the validated guides and scales that exist for their detection. Conclusions: The identification of risk behaviors and early intervention in adolescents decrease their morbidity and mortality. It is recommended that health professionals use the best available screening tools such as Home/environment, Education/employment, peer Activities, Drugs, Sexuality and Suicide/depression (HEADDS); Rapid Assessment for Adolescent Preventive Services (RAAPS); and Global school-based student health survey (GSHS). These guides quickly and simultaneously assess multiple behaviors, are easily applied in primary care, and facilitate the approach and management of this population.
\end{abstract}

Keywords: Adolescent; adolescent behavior; health risk behaviors; prevalence; protective factors; risk assessment. (Source: DeCS, Bireme).

*Autor de correspondencia Luz Adriana Patiño Guerrero e-mail: luzpagu@unisabana.edu.co 


\section{Introducción}

La adolescencia es una etapa crítica del desarrollo por cuanto la vulnerabilidad y los múltiples cambios a los que se somete el individuo afectan su personalidad y sus actos de la vida cotidiana. En esta época se consolidan los hábitos relacionados con el cuidado de la salud y el bienestar que dependen de las oportunidades con que dispone el individuo, como acceso a la educación y lazos familiares, entre otros $^{(1)}$. El consumo de sustancias psicoactivas (SPA), prácticas sexuales de riesgo y problemas de salud mental se consideran los principales comportamientos de riesgo en los adolescentes. $\mathrm{Su}$ correcta medición permite entender la problemática para diseñar intervenciones que impacten en los determinantes del riesgo(2). A pesar de esta recomendación, se encuentra que el abordaje del riesgo es bajo (entre 3\% a 25\%)(3). A lo largo de la historia se han realizado diferentes intervenciones psicosociales para el manejo de los comportamientos de riesgo en este grupo; sin embargo, para que generen impacto, se requiere de un seguimiento en el tiempo, ya que la mayoría de los estudios en esta población son a corto plazo(4).

El comportamiento de riesgo en salud de los adolescentes, se define como toda actividad que aumente la probabilidad de perder la condición de salud y que contribuye a una muerte prematura o discapacidad(5). Estos comportamientos son dinámicos y cambiantes en el tiempo y el espacio(6). En Estados Unidos, la Iniciativa Nacional para Mejorar la Salud de los Adolescentes clasificó los comportamientos riesgosos en cinco dominios a saber: violencia, consumo de sustancias, salud mental, salud sexual y hábitos inadecuados que predisponen al desarrollo de enfermedades crónicas.

Se han descrito factores protectores que disminuyen la probabilidad de alteración del comportamiento y factores de vulnerabilidad que facilitan conductas de riesgo. Estos se encuentran organizados en tres categorías: Factores intrapersonales, interpersonales y culturales/ambientales. Los intrapersonales son las cualidades innatas de cada individuo que están determinadas por la genética, rasgos físicos y de personalidad, desarrollo puberal, género, tendencias afectivas y capacidad mental. Por su parte, los factores interpersonales se refieren a la relación que existe entre el adolescente y otros individuos, y las consecuencias que se producen de esta interacción con padres, profesores, pares entre otros; de la calidad de esta relación dependerá la probabilidad de que el adolescente presente conductas de riesgo. Finalmente, el componente cultural representa la interacción entre los factores inter e intrapersonales con el entorno ${ }^{5}$. Por lo anterior, el objetivo de esta revisión fue identificar los principales comportamientos de riesgo de los adolescentes, su prevalencia, factores predisponentes, así como las guías y escalas validadas que existen para su oportuna detección.

\section{Materiales y métodos}

La búsqueda bibliográfica se realizó en las bases de datos PubMed, ClinicalKey, SciELO, ScienceDirect y páginas oficiales de la Organización Mundial de Salud (OMS), el Centro de Control de Enfermedades (CDC) por sus siglas en inglés y la Organización Panamericana de Salud (OPS).

De 237 referencias encontradas se seleccionaron sesenta y cinco documentos entre artículos completos y reportes de páginas web, que cumplieron con los siguientes criterios de inclusión: 1. Idioma español o inglés, 2. Haber sido publicados entre los años 2003 y 2019, excepto una cita bibliográfica del año 1999 para describir la fecha de la creación de la encuesta SCOFF, útil en trastornos de la conducta alimentaria en adolescentes. Se excluyeron aquellos artículos escritos en otros idiomas y publicados antes del año 2003 con la excepción ya mencionada. Como términos de búsqueda se utilizaron en español: adolescente, comportamiento del adolescente, conductas de riesgo para la salud, prevalencia, factores protectores, evaluación de riesgo; y en inglés: adolescent, adolescent behavior, health risk behavior, prevalence, protective factors, risk assessment. Se utilizaron los conectores booleanos AND, OR y NOT. Los artículos encontrados se clasificaron y agruparon según las cinco categorías de comportamientos de riesgo en población adolescente ya descritas, y se realizaron resúmenes con datos de prevalencias a nivel global y local, así como de los métodos validados para su detección.

\section{Resultados}

Para describir la prevalencia de los comportamientos de riesgo se agrupó la información en los 5 dominios a saber: 


\section{Violencia y lesiones no intencionales}

Violencia según la OPS es "el uso intencional de la fuerza o el poder físico, de hecho, o como amenaza contra uno mismo, otra persona, un grupo o comunidad, que cause o tenga muchas probabilidades de causar lesiones, muerte, daños psicológicos, trastornos del desarrollo o privaciones"(7). En el mundo el $71 \%$ de los adolescentes mueren por causas externas como accidentes de tránsito, homicidio y suicidio($^{(8)}$. La OMS reportó que en 2015, 1,2 millones de adolescentes en el mundo murieron por causas prevenibles(9). Asimismo, cada año se producen alrededor de 200.000 homicidios en adolescentes y adultos jóvenes y de estos el 83\% son hombres(7). Otra forma de violencia, definida por el Center for Disease Control and Prevention (CDC) es el acoso escolar, que se refiere a uno o más estudiantes que bromean, amenazan, difunden rumores, agreden físicamente, empujan o lastiman a otro estudiante de forma repetida. En el estudio de Redfield et al., en Estados Unidos el 19\% de los estudiantes refirió haber sido víctima del matoneo dentro de las instituciones educativas, en los 12 meses previos a la aplicación de la encuesta(10).

En el año 2010, la OPS reportó para la Región de las Américas 287.920 defunciones secundarias debido a traumatismos, actos violentos $\mathrm{y}$ homicidios de jóvenes entre los 15 y 19 años(2). En Colombia, el Instituto Nacional de Medicina Legal y Ciencias Forenses (INMLCF) en el año 2016, registró 10.435 casos de violencia intrafamiliar en niños/as y adolescentes ${ }^{(11) .}$

\section{Consumo de sustancias}

El modelo que explica el uso de sustancias es complejo, por cuanto intervienen diversos factores: genético-ambientales, trastornos psiquiátricos, experiencias previas, la influencia de la familia y sus pares, el colegio, trabajo, religión y realización de algún deporte(12). La OMS informó que en el año 2010 el 38\% de la población mayor de 15 años había consumido alcohol en los últimos 12 meses(13). En Estados Unidos, el Youth Risk Behavior Surveillance System (YRBSS) del CDC - 2017 reportó que en los 30 días previos el 29,8\% de los estudiantes había bebido alcohol, el 14\% había fumado cigarrillo y el 19,8\% usó marihuana. Respecto a las demás drogas ilícitas, el 6,6\% las consumió una o más veces durante su vida(10).
Acorde con reportes de la OPS en la región de las Américas, 1 de cada 10 jóvenes de 16 a 18 años consumía 4 o más bebidas alcohólicas a la vez, y aproximadamente 1 de cada 10 adolescentes de 13 a 17 años consumió drogas ilícitas por lo menos una vez en su vida, siendo la marihuana la más consumida. El consumo de tabaco en el último mes osciló entre 2,2\% y 38,7\% en los diferentes países latinoamericanos(14). El Análisis de Situación de Salud (ASIS) 2015 en Colombia señaló que el consumo de SPA, alcohol y tabaco viene en ascenso. La edad de inicio promedio es de 12 años para alcohol con mayor prevalencia en estratos altos. Con respecto al uso de sustancias, el consumo más alto se presentó entre 18 y 24 años $(49,2 \%)$ de los encuestados. Al igual que en la mayoría de países, la marihuana, es la droga ilícita de mayor consumo en el Colombia, $62 \%$ más frecuente en hombres, con una edad de inicio de 14 años. El 42\% de los encuestados ha consumido tabaco en algún momento de su vida con una mayor prevalencia en hombres (71\%)(11).

\section{Salud mental}

En la etapa de adolescencia las patologías mentales constituyen la primera causa de carga de enfermedad puesto que tienden a ser crónicas y se asocian a mal pronóstico si emergen a temprana edad(15). La OMS indicó que la mitad de los trastornos de salud mental en la adultez iniciaron a los 14 años; sin embargo, no fueron adecuadamente diagnosticados ni tratados(9). El YRBSS/CDC-2017, informó que en el año previo a la realización del estudio, el 31,5\% de los estudiantes se sintió triste y sin esperanza casi a diario por 2 o más semanas y el $17,2 \%$ habrían considerado suicidarse, con una prevalencia de $22,1 \%$ en mujeres y $11,9 \%$ en hombres(10).

Existe una prevalencia entre el 18\% y el 25\% de trastornos mentales en América Latina y el Caribe a nivel comunitario, y entre un $27 \%$ a $48 \%$ en el ámbito clínico. De estos entre 12\% y $29 \%$ corresponden a niños y adolescentes(2). En Colombia, según la Encuesta Nacional de Salud Mental 2015, la prevalencia para los trastornos mentales fue del 17\%; y dentro de los más frecuentes se registraron: trastornos de ansiedad (agorafobia 4,5\% y fobia social 1,5\%), trastornos de conducta disruptiva $3,3 \%$, trastornos por consumo de sustancias (abuso y/o uso de marihuana 3,4\% y alcohol 2,9\%) y depresión mayor con una prevalencia del 1,7\%(15). 


\section{Salud sexual}

La sexualidad constituye uno de los aspectos importantes en el desarrollo de un adolescente, es un continuo de comportamientos y actitudes que están motivados por el deseo sexual, y encaminados hacia la gratificación y el placer(16). En 2018 la OMS señaló que existen 2,1 millones de adolescentes con virus de inmunodeficiencia humana VIH en el mundo(9). Los adolescentes europeos continúan siendo un grupo vulnerable para la adquisición de enfermedades de transmisión sexual (ETS); la proporción de hombres con 2 o más parejas sexuales es del $50 \%$ en países como Finlandia y España, mientras que las mujeres entre 15 y 16 años con la mayor proporción corresponden a Finlandia (54\%) ${ }^{(17) .}$

El YRBSS/CDC-2017 mostró que en Estados Unidos el 39,5\% de los estudiantes han tenido al menos un encuentro sexual, con mayor prevalencia en hombres que en mujeres $(41,4 \%$ vs $37,7 \%$ respectivamente). En el mismo reporte se informó que ocurrieron 488.700 casos de ETS (clamidia, gonorrea y sífilis) y 1.652 diagnósticos de VIH en la población adolescente(10). En América Latina y el Caribe los hombres tienen su primera relación sexual entre los 12,7 años (Jamaica) y 16 años (Chile). Las mujeres por su parte tienen un rango de edad que varía entre los 15,6 y 17,9 años en Jamaica y Chile respectivamente(16). Las ETS, incluyendo el VIH/SIDA, en América Latina y el Caribe se presentan especialmente en mujeres que viven en condiciones de pobreza y donde las relaciones sexuales transaccionales son habituales(2).

Según la Encuesta Nacional de Demografía y Salud (ENDS 2015), en Colombia la edad mediana de la primera relación sexual es de 17,7 años en mujeres y 16,1 años en hombres; el 16,3\% de las mujeres y el $33,1 \%$ de los hombres de 15 a 24 años tuvieron su primera relación sexual antes de los 15 años(18). En el país la prevalencia de VIH/SIDA entre los 15 y 49 años ha disminuido de $0,7 \%$ en el año 2006 a 0,52\% en 2011 de acuerdo con el Plan Decenal de Salud Pública (19); y a 0,48\% en el 2015. (20). Según informe del Ministerio de Salud y Protección Social, durante el periodo 2009-2011 se reportó un promedio anual de 94.000 casos de ETS, con una mayor frecuencia en mujeres y en el grupo de 20-29 años de edad(21).

Otro de los problemas relacionados con los comportamientos sexuales de riesgo es el embarazo precoz, que ocurre entre los 13 y 19 años. Los embarazos se presentan en $25 \%$ de las adolescentes a nivel mundial y en un 13\% en Estados Unidos; y de estos el $80 \%$ no planeados ${ }^{(22) .}$

En la Región de las Américas, la mitad de los países presentan tasas de fecundidad entre adolescentes de 15 a 19 años superiores a 72 por 1000 y el $20 \%$ de los partos correspondió a mujeres menores de 20 años (14). En Colombia la fecundidad entre 15 a 19 años ha disminuido, encontrándose un 17,4\% de mujeres madres o embarazadas del primer hijo, en comparación con el 19,5\% en el 2010. El mayor descenso se dio en adolescentes con mejores condiciones socio-económicas(18).

\section{Hábitos inadecuados y desarrollo de enfermedades crónicas}

Como parte fundamental del desarrollo de los niños y durante su etapa de crecimiento hacia la adolescencia deben existir hábitos saludables de nutrición balanceada y ejercicio regular, pues de estos depende un crecimiento adecuado, desarrollo de funciones corporales, así como de las habilidades cognitivas y sociales, lo cual contribuye en la prevención de enfermedades crónicas en la adultez $^{(23)}$. El reporte del YRBSS/CDC-2017 en Estados Unidos, informó que, durante los 7 días previos a la encuesta, el 5,6\% y el 7,2\% de los adolescentes no consumieron frutas ni vegetales respectivamente. Con respecto a la actividad física el $15,4 \%$ de los adolescentes no participó durante mínimo 60 minutos, en alguna actividad moderada a vigorosa en al menos 1 de los últimos siete días anteriores a la encuesta(10). Los datos en Colombia según la Encuesta Nacional de la Situación Nutricional (ENSIN) 2015, mostraron que el cumplimiento de recomendaciones de actividad física en adolescentes fue mayor en hombres $(18,7 \%)$ en comparación con las mujeres $(7,6 \%)^{(24)}$.

En cuanto a los problemas de salud derivados de hábitos inadecuados de alimentación y actividad física se destacan los siguientes:

Trastornos de la conducta alimentaria: Son patologías caracterizadas por actitudes y comportamiento anormales frente al consumo de alimentos, alteraciones psicológicas, distorsión de la percepción corporal e incluso temor intenso a la obesidad(25). La prevalencia de estos trastornos en adolescentes oscila entre $1 \%$ y $8 \%$ en países 
industrializados (26). Estos desórdenes se relacionan con baja autoestima y depresión(27).

Malnutrición y obesidad: Según la OMS estas entidades presentan un impacto considerable en la salud pública. La desnutrición en niños que llegan a la adolescencia, puede hacerlos más propensos a contraer padecer otro tipo de enfermedades, o morir en edad temprana. Por su parte, la obesidad viene en aumento en los países de altos y bajos ingresos produciendo a su vez un mayor riesgo cardiovascular en esta población( ${ }^{(9)}$.

Para el año 2015 en Norteamérica se encontró que el $13,9 \%$ de jóvenes padecen de obesidad, con una prevalencia de $16,8 \%$ en hombres y de $10,8 \%$ en mujeres; así como una mayor frecuencia entre los estudiantes afroamericanos (16,8\%) e hispanos $(16,4 \%)$, en comparación con los de raza blanca $(12,4 \%)^{(8)}$. En Colombia la ENSIN 2015 encontró que las mujeres adolescentes tienen un $21,2 \%$ de sobrepeso en comparación con los hombres $(14,8 \%)$ y de manera preocupante el exceso de peso en adolescentes (13 a 17 años) aumentó 2,4 puntos porcentuales entre $2010(15,5 \%)$ y $2015(17,9 \%)^{(24)}$.

\section{Escalas y herramientas para detectar comportamientos de riesgo en adolescentes}

Dentro de las escalas integradas validadas para evaluar comportamientos de riesgo a nivel mundial se encuentra la "Encuesta Mundial de Salud en Escolares" (GSHS) por sus siglas en inglés, fue creada por la OMS, entidad que desde 2003 junto en la OPS y en coordinación con el CDC de Atlanta y Ministerios de Salud y Educación de cada región colabora con la implementación de la encuesta en cada uno de los países de las Américas. Esta herramienta permite evaluar los factores de riesgo para el consumo de alcohol, comportamiento alimentario, consumo de drogas, salud mental, actividad física, comportamientos sexuales, tabaco y violencia; así como factores protectores para estas mismas categorías agrupados en 10 módulos(28-30).

La encuesta mundial se aplicó por primera vez en algunas ciudades de Colombia en el año 2018, en jóvenes entre 13 y 17 años. Se denominó "Encuesta Nacional de Salud Escolar" (ENSE) y sus resultados permitieron evaluar las prioridades, generar programas de salud nacional y comparar los resultados con los demás países del mundo donde se han $\operatorname{aplicado}^{(31) .}$
En Estados Unidos, el YRBSS fue creado en 1990 como una iniciativa del CDC, para monitorizar los comportamientos en salud de estudiantes entre noveno y décimo segundo grado y evalúa las principales causas de muerte y discapacidad entre jóvenes, a través de la evaluación de 6 categorías: Comportamientos que contribuyen a las lesiones involuntarias y violencia, comportamientos sexuales relacionados con embarazos no deseados y ETS, incluida la infección por VIH, alcohol y otras drogas, consumo de tabaco, comportamientos dietéticos poco saludables y actividad física inadecuada(32).

Las directrices de las "Guías de Servicios Preventivos para Adolescentes" (GAPS) por sus siglas en inglés se desarrollaron por la American Medical Association (AMA) en 1993 para identificar los comportamientos de riesgo más prevalentes en adolescentes, mediante la elaboración de cuestionarios para ser aplicados en adolescentes y sus padres(33).

Esta herramienta ha sido considerada el "Gold standard" en la evaluación de los riesgos para la salud de los adolescentes; pero su aplicación resulta dispendiosa ya que consta de 60 a 71 preguntas y requiere tiempo para su ejecución. Por esta razón, la AMA permitió una modificación para realizar una versión simplificada, que se desarrolló por la Universidad de Michigan en el año 2009, denominada "Evaluación Rápida para Servicios Preventivos para Adolescentes" (RAAPS) por sus siglas en inglés, la cual comprende 21 preguntas relativas a los cinco dominios de comportamientos de riesgo ya mencionados, y se puede utilizar en ámbitos clínicos y escolares. Su versión en inglés tiene adecuados índices de validez(34). La versión en español fue recientemente validada en Colombia, encontrando una validez aceptable pero limitada con mayor utilidad en la detección de conductas de riesgo para consumo de alcohol, sustancias psicoactivas y tabaco(35).

Una de las recomendaciones clínicas en la atención ambulatoria de los adolescentes es la entrevista psicosocial mediante el uso del acrónimo (HEADSS) que se deriva de las palabras: Home, Education, Activities, Drugs, Sex and Suicide. Se sugieren 67 preguntas abiertas para abarcar estos temas y así evaluar el comportamiento de los adolescentes(36). La Academia Americana de Pediatría (AAP) a partir del año 1990 desarrolló las guías "Bright Futures", para la promoción de la salud y prevención de las 
enfermedades en niños y adolescentes, a través de recomendaciones de tamizaje y guías anticipatorias en las diferentes etapas desde la consulta prenatal hasta el final de la adolescencia a los 21 años(36).

Otra escala importante es la Escala de Conductas de Riesgo en Adolescentes (ECRA), la cual fue adaptada del cuestionario "Adolescent Health Survey", creado por Robert Bum y colaboradores en la universidad de Minesotta en 1997 y validada al idioma español en Chile en el año 2006(37). En Colombia, el Departamento Administrativo Nacional de Estadística (DANE) a partir del año 2006 implementó la "Encuesta de Comportamientos y Factores de Riesgo en Niñas, Niños y Adolescentes Escolarizados" (ECAS) para ser aplicada en estudiantes de bachillerato, con el objetivo de determinar múltiples conductas de riesgo, factores sociales, individuales y familiares que favorecen la explotación sexual en población infantil y adolescente, así como las consecuencias del abuso(38). Además de las herramientas para detectar simultáneamente múltiples riesgos ya mencionadas, existen escalas o cuestionarios específicos para la evaluación de cada una de las cinco categorías de riesgo en la población juvenil:

Violencia: Para evaluar este dominio, en cuanto al acoso escolar, existe el Cuestionario de Bullying de Olweus (OBQ) que permite realizar un análisis psicométrico del matoneo. El cuestionario ha sido ampliamente validado y aplicado a nivel mundial(39). La escala Child Adolescent Bullying Scale (CABS), permite identificar a jóvenes en riesgo de acoso, consta de 22 ítems y está desarrollada específicamente para evaluarlo según la definición del CDC(40).

Se han diseñado otros instrumentos para evaluar el acoso escolar, como la escala de Cisneros de Piñuel y Oñate(41), validada en Colombia en 2008; el Cuestionario de intimidación escolar y la "Encuesta de Clima y Seguridad Escolar de California" (CSCSS), un instrumento de 15 ítems desarrollado por Rebelez y Furlong, que contiene categorías que permiten un análisis de la violencia o las agresiones en las instituciones educativas, convivencia escolar entre estudiantes y profesores, conflictos y la victimización. Todos ellos presentan una adecuada fiabilidad para su uso en el entorno clínico(42). Adicionalmente, la "Escala de Tácticas de Conflicto Modificada", la cual evalúa la persistencia y victimización de conductas violentas tanto psicológicas y físicas en las relaciones de pareja entre adolescentes ${ }^{(43)}$.

Consumo de sustancias: Para la detección del consumo de alcohol o drogas se implementó la escala CRAAFT, creada por Knight, et al., en Estados Unidos, la cual es fácil de utilizar y está validada en adolescentes(44). Consta de 6 preguntas que evalúan si ha conducido en estado embriaguez o bajo efecto de alguna SPA, su uso como medio para relajarse, si amigos o familiares le han sugerido que disminuya el consumo, si ha consumido a solas, si ha tenido problemas al estar bajo efecto de estas sustancias o si ha olvidado lo que hizo mientras las consumía. Dos o más respuestas positivas alertan al profesional para realizar una evaluación exhaustiva en el tema. Esta escala fue validada en Colombia en jóvenes entre 14 y 18 años de edad para la identificación de consumo disfuncional(45).

Dentro de la categoría de consumo indebido de alcohol existen otras herramientas de análisis tales como: el "Inventario de consumo de Adolescentes" que consta de 24 ítems que refleja el perfil del adolescente y su falta de control por la bebida; el "Índice de Problemas de Alcohol Rutgers" que demuestra el área funcional afectada por el consumo de alcohol y la "Escala de Trastorno Obsesivo Compulsivo del Adolescente" que relaciona los comportamientos compulsivos con el consumo de bebidas embriagantes ${ }^{(12)}$.

El cuestionario "CAGE", se utiliza para medir dependencia alcohólica o consumo abusivo de acuerdo a las cuatro preguntas que se evalúan: Crítica social, culpa individual, ingesta matutina y necesidad sentida por dejar de consumir alcohol(46). Fue validado para Colombia entre el año 2002 y 2003 con un índice kappa entre moderado y bueno(47).

Salud mental: La herramienta de elección para la detección de desórdenes mentales es el Child Behavior Checklist (CBCL), creado por Achenbach en el año 1991, que permite tamizar a niños y adolescentes entre 4 y 18 años con problemas comportamentales $\mathrm{y}$ emocionales $\mathrm{y}$ puede ser desarrollado por su familia durante 30 a 60 minutos(48). Para analizar los síntomas de depresión en jóvenes se diseñó una escala de 20 preguntas denominada "Centro de Estudios Epidemiológicos, Escala de Depresión” (CES-D) por sus siglas en inglés, 
descrita por Radloff en 1977; donde se evidencia la frecuencia de la presentación de los síntomas en los últimos 7 días(49). De acuerdo con el boletín de salud mental del Ministerio de Salud, en Colombia existe el Cuestionario de Reporte de Niños (RQC), que permite la detección de síntomas sugestivos de problemas mentales $(50,51)$.

\section{El "Screen for Child Anxiety Related Disorders"} SCARED, contiene 41 ítems, se aplica en población entre 9 a 18 años en donde cada ítem consta de tres afirmaciones y se usa para la tamización de síntomas sugestivos de ansiedad. Por su parte, el "Children's Depresion Inventory" (CDI) es una de las herramientas más utilizadas para identificar sintomatología que sugiera depresión en los adolescentes. Ambas escalas se utilizaron en Colombia en un estudio para determinar prevalencia de síntomas ansiosos y depresivos en estudiantes de 10 a 17 años de Chía, Cundinamarca(52).

Salud sexual: Una de las herramientas disponibles para medir las actitudes sexuales es la Encuesta Revisada de Opinión Sexual (EROS), validada por Carpintero y Fuertes en España. En 2017 se publicó su versión reducida, la cual por medio de 8 preguntas permite identificar si la actitud del adolescente es positiva o negativa frente a material de contenido sexual, onanismo y erotismo(53).

Bello-Villanueva et al., diseñaron y validaron una escala de intención de protección y de riesgo en sexualidad reproductiva entre adolescentes escolares de 11 a 19 años de ambos sexos en el Caribe colombiano. Dicha escala consta de 6 ítems basados en actitudes personales, creencias normativas y de control percibido entre otros; y evalúa la intención de las adolescentes mujeres y varones para utilizar métodos de protección, abstinencia y retardo en el inicio de la actividad sexual, o prevención de embarazos tempranos y ETS en el caso hipotético de planear y/o estar dispuesto a tener relaciones sexuales. Los ítems de intención hacia comportamientos sexuales y reproductivos se organizaron en dos grupos: intención de riesgo e intención hacia la protección para cada uno de los géneros. Se evalúa en un formato de respuesta tipo likert con puntuaciones de 1 a 5 y con alternativas que fluctúan desde totalmente en desacuerdo hasta totalmente de acuerdo (54).
Hábitos inadecuados que predisponen al desarrollo de enfermedades crónicas: Para abordar los trastornos de la conducta alimentaria en adolescentes se creó la encuesta SCOFF (del acrónimo en inglés sick, control, one, fat, food), que consiste en un cuestionario sencillo de 5 preguntas que evalúan las características principales de anorexia y bulimia nerviosa: se considera caso probable si se presenta un puntaje de 2 respuestas afirmativas(55). SCOFF fue traducido al español y validado en mujeres colombianas entre los 17 y 35 años, es una herramienta adecuada para tamizar poblaciones grandes dada su sencillez y rapidez de administración(56).

El Eating Attitudes Test (EAT) fue diseñado por Garner y Garfinkel en 1979, es un cuestionario de actitudes ante la alimentación para la evaluación del comportamiento en anorexia. En contraste el Bulimic Investigatory Test Edimburgo (BITE), diseñado por Henderson et al., (57) permite evaluar sintomatología bulímica en los últimos tres meses previos a la prueba, a partir de 33 ítems agrupados en dos subescalas. La puntuación máxima es de 39 puntos y sus síntomas se agrupan en: sin riesgo, situación de riesgo y desorden de alimentación(58).

El "Cuestionario Breve para Medir Conductas Alimentarias de Riesgo" se desarrolló con base en los criterios del Diagnostic and Statistical Manual, 4th edition (DSM - IV); y evalúa la preocupación por aumentar de peso, atracones, falta de control con la ingesta de alimentos, conductas restrictivas (dietas, ayuno, ejercicio y uso de fármacos para perder peso) y purgativas (emesis autoinducida, uso de laxantes y diuréticos). El formato consta de 11 preguntas que revelan la conducta de riesgo en los 3 meses previos a la aplicación del instrumento(59,60). El HBSCSymptom Checklist es un cuestionario con 8 ítems creado para el estudio Health Behavior in Schoolaged Children (HBSC), en colaboración con la OMS para evaluar los comportamientos saludables, estilo de vida y la percepción de salud por los adolescentes(61). Dentro del cuestionario se incluye información de los factores demográficos, el contexto sociocultural, los desenlaces en salud, hábitos saludables (como el tipo de alimentación, actividad física y la pérdida de peso) así como hábitos de riesgo (consumo de cigarrillo, alcohol y otras sustancias, comportamiento sexual inadecuado y matoneo) ${ }^{(62) . ~ L o s ~ c u e s t i o n a r i o s ~ d e ~ f r e c u e n c i a ~ d e ~}$ consumo (FFQ) permiten identificar la relación entre 
la dieta y la enfermedad o factores de riesgo para desarrollar una patología determinada de acuerdo a la lista de alimentos, frecuencia de consumo y tamaño de la porción consumida. Sus ventajas radican en que pueden realizarse personalmente $o$ vía telefónica. Si se desarrolla por menores de edad o personas con discapacidad, los padres o cuidadores deben estar presentes al momento de realizarlos ${ }^{(63)}$.

\section{Discusión}

La variabilidad encontrada en las cifras de prevalencia se podría explicar por las diferencias en las características sociodemográficas de la población estudiada y la precisión de la herramienta de tamización utilizada, en términos de sensibilidad y especificidad. La implementación de las diferentes escalas requiere de una rigurosidad estadística en cuanto a sensibilidad y reproducibilidad, así como un adecuado sistema de validación en la población objeto, lo cual desafortunadamente no se cumple en todos los casos $(2,3,4)$

En el año 2013 el Banco Interamericano de Desarrollo (BID), publicó la guía para medir comportamientos de riesgo en jóvenes la cual sugiere pautas para el diseño de instrumentos que midan dichas conductas, con énfasis en la sensibilidad de los datos, privacidad y manejo confidencial de los mismos. Se desarrolla en capítulos que abordan temáticas como la calidad de la información, el diseño de instrumentos, conductas y factores de riesgo a medir, confiabilidad de los datos, organización del trabajo de campo y costoefectividad de la aplicación de las encuestas(2).

En Estados Unidos se implementó desde la década de los noventa por parte del CDC un Sistema de Vigilancia de Comportamientos de riesgo en Jóvenes, con reportes unificados cada 2 años de una muestra representativa de escolares de todo el país. De esta manera han logrado crear políticas de prevención y medir su efectividad en el tiempo( ${ }^{(8)}$.

La OMS ha desarrollado algunas herramientas para detectar conductas y factores de riesgo en jóvenes escolares de las diferentes regiones del mundo ${ }^{9}$. En la práctica se realizan en algunos países estudios piloto de forma fraccionada, utilizando la escala mundial de la OMS, con muestras algunas veces no lo suficientemente representativas y sin un seguimiento en el tiempo, que permita establecer un sistema global de vigilancia periódica de los comportamientos de riesgo en la población adolescente $(4,30)$

Así como en muchos países, en Colombia no se cuenta con un modelo unificado de reporte periódico de dichas conductas, que permita conocer su frecuencia para poder de esta manera implementar políticas y estrategias efectivas en la prevención primaria y secundaria priorizando en el abordaje de los riesgos de mayor prevalencia.

Como recomendaciones se sugiere, de acuerdo con lineamientos de entes reguladores globales y locales, la creación e implementación de escalas multirriesgo validadas, que sean cortas, amigables y útiles en la detección idónea de conductas de riesgo en jóvenes en un ámbito de atención primaria tanto clínico como educativo. De esta manera y según hallazgos se podrán realizar intervenciones oportunas y estrategias de prevención contando con la participación activa de los diferentes actores de la sociedad $(64,65)$.

Una de las principales limitaciones para el abordaje de los peligros a los que se exponen los adolescentes es lograr la creación de herramientas que tengan adecuada sensibilidad y especificidad para que los datos sean confiables. Las herramientas disponibles para la detección de conductas de riesgo en jóvenes son múltiples y no todas las escalas están validadas para todo tipo de población, lo que dificulta su aplicabilidad y reproducibilidad. Por otra parte, se requiere del consentimiento firmado por los padres de los participantes que sean menores de edad, no todas son anónimas y algunas son realmente extensas, lo que hace dispendiosa su aplicación. No hay garantía en la veracidad de datos ya que no existe forma de proteger la confidencialidad de forma absoluta, pues prima la integridad del adolescente sobre la privacidad en las respuestas de las pruebas, cuando se detecten situaciones que se consideren de alto riesgo (64). Lo ideal sería que las escalas y documentos fueran anónimos, sin información relacionada con la identificación, lo cual sigue siendo un desafío porque existe responsabilidad en el médico o profesional de salud que la realice, incluso de tipo médico legal. Para esto se sugiere que el manejo este a cargo de un equipo multidisciplinario, que brinde respeto, confianza, sin discriminación alguna y un ambiente propicio para que el joven se sienta a gusto con las respuestas de cada una de las 
pruebas que se le practiquen (2,4). Además, que se permita privacidad en el momento de contestar los cuestionarios, sin que estén presentes los padres ni los encuestadores y mucho menos se realicen preguntas diferentes al tema que se esté evaluando con el menor(2).

\section{Conclusiones}

Los comportamientos de riesgo para la salud en los adolescentes son variables en su frecuencia $y$ dependen para su desarrollo, del equilibrio entre factores protectores y precipitantes. Aunque se han propuesto múltiples escalas para la medición aislada o integrada de los 5 dominios de riesgo en jóvenes; se requiere la implementación de sistemas unificados de vigilancia mediante el uso de herramientas tales como: El acrónimo HogarEducación-Actividades-Drogas-Sexualidad-Suicidio (HEADSS), el cuestionario Rapid Assessment for Adolescent Preventive Services (RAAPS) o la encuesta Global school-based student health survey (GSHS) que sean confiables, validadas, amigables, costo-efectivas $\mathrm{y}$ reproducibles en las diferentes poblaciones.

Los profesionales de la salud deben estar entrenados para abordar de manera integral estos comportamientos, ya que la prevención y detección oportuna de los mismos disminuye los costos de salud y reduce la morbi-mortalidad en este grupo etario.

\section{Agradecimientos}

Los autores agradecen a la Universidad de La Sabana por su valiosa contribución para el acceso a las bases de datos.

Conflictos de interés: Los autores declaran no tener ningún conflicto de interés.

\section{Referencias}

1. Patton GC, Sawyer SM, Santelli JS, Ross DA, Afifi R, Allen NB, et al. Our future: a Lancet commission on adolescent health and wellbeing. The Lancet. 2016;387(10036):2423-78. https://doi.org/10.1016/S0140-6736(16)00579-1

2. Bautista-Arredondo S, -Peña P, McCoy S, Muñoz R, Saric D, Vivo S. Guía para medir comportamientos de riesgo en jóvenes. BID; 2013. Disponible en: http://www.codajic.org/sites/www.codajic.org/files/Guia \%20medicion\%20comportamiento\%20riesgo\%20jovenes. pdf

3. Kadivar H, Thompson L, Wegman M, Chisholm T, Khan M, Eddleton $\mathrm{K}$, et al. Adolescent Views on Comprehensive Health Risk Assessment and Counseling: Assessing Gender
Differences. J Adolesc Health. julio de 2014;55(1):24-32. https://doi.org/10.1016/j.jadohealth.2013.12.002

4. Salam RA, Das JK, Lassi ZS, Bhutta ZA. Adolescent Health Interventions: Conclusions, Evidence Gaps, and Research Priorities. J Adolesc Health. 2016;59(4):S88-92. https://doi.org/10.1016/j.jadohealth.2016.05.006

5. Keeler HJ, Kaiser MM. An Integrative Model of Adolescent Health Risk Behavior. J Pediatr Nurs. 2010;25(2):126-37. https://doi.org/10.1016/j.pedn.2009.01.005

6. Meneses C, Romo N, Uroz J, Gil E, Markez I, Giménez S, et al. Adolescencia, consumo de drogas y comportamientos de riesgo: diferencias por sexo, etnicidad y áreas geográficas en España. Trastor Adict. 2009;11(1):51-63. https://doi.org/10.1016/j.pedn.2009.01.005

7. Organización Panamericana de la Salud, Organización Mundial de la Salud. La prevención de la violencia juvenil: panorama general de la evidencia [Internet]. Ginebra: OPS/OMS; 2016 [citado 23 de febrero de 2018]. Disponible en:

iris.paho.org/xmlui/bitstream/handle/123456789/28248 /9789275318959_spa.pdf?sequence $=5$ \&isAllowed =y

8. Kann L, McManus T, Harris WA, Shanklin SL, Flint KH, Hawkins J, et al. Youth Risk Behavior Surveillance-United States, 2015. Morbidity and Mortality Weekly Report. Surveillance Summaries. Cent Dis Control Prev. 2016;65(6):1-174. Disponible en https://www.cdc.gov/mmwr/volumes/65/ss/ss6506a1.ht $\mathrm{m}$

9. Organización Mundial de la Salud. Adolescentes: riesgos para la salud y soluciones [Internet]. Ginebra: OMS; 2017 [citado 23 de febrero de 2018]. Disponible en: http://www.who.int/mediacentre/factsheets/fs345/es/

10. Redfield R, Schuchat A, Dauphin L. Youth Risk Behavior Surveillance, Morbidity and Mortality Weekly Report. United States; 2017. (Morbidity and Mortality Weekly Report). Cent Dis Control Prev. 2018;67(8):1-114. Disponible en: https://www.cdc.gov/mmwr/volumes/67/ss/ss6708a1.ht m

11. Ministerio de Salud. Análisis de situación de salud (ASIS) Colombia [Internet]. MinSalud: Bogotá; 2016 [citado 23 de febrero de 2018]. Disponible en: https://www.minsalud.gov.co/sites/rid/Lists/BibliotecaDi gital/RIDE/VS/ED/PSP/asis-colombia-2016.pdf

12. Margret CP, Ries RK. Assessment and Treatment of Adolescent Substance Use Disorders: Alcohol use disorders. Child Adolesc Psychiatr Clin N Am. 2016;25(3):411-30. https://doi.org/10.1016/j.chc.2016.03.008

13. Llopis EJ. Funding mechanisms for the prevention and treatment of alcohol and substance use disorders [Internet]. España: ESADE Business School, Ramon Llull University; 2017 [citado 22 de febrero de 2018]. Disponible en: www.who.int/substance_abuse/activities/fadab/msb_adab _funding.pdf

14. Organización Panamericana de la Salud. Estrategia y plan de acción regional sobre los Adolescentes y Jóvenes 2010 2018 [Internet]. Washington: OPS; 2010 [citado 23 de febrero de 2018]. Disponible en: http://new.paho.org/hq/dmdocuments/2011/Estrategiay-Plan-de-Accion-Regional-sobre-los-Adolescentes-yJovenes.pdf

15. Gómez-Restrepo C, Bohórquez A, Tamayo Martínez N, Rondón M, Bautista N, Rengifo $\mathrm{H}$, et al. Trastornos depresivos y de ansiedad y factores asociados en la 
población de adolescentes colombianos, Encuesta Nacional de Salud Mental 2015. Rev Colomb Psiquiatr. 2016;45:50-7. https://doi.org/10.1016/j.rcp.2016.09.009

16. Vargas E, Ponsoda V. Escala de actividad sexual: un instrumento para predecir el inicio temprano de relaciones sexuales. Psicol Conduct. 2010;18(3):591-611.

17. Calatrava M, López-Del Burgo C, de Irala J. Factores de riesgo relacionados con la salud sexual en los jóvenes europeos. Med Clínica. 2012;138(12):534-40. https://doi.org/10.1016/j.medcli.2011.07.020

18. Profamilia, Ministerio de Salud. Encuesta Nacional de Demografía y Salud, Colombia 2015. Tomo II. 2016. p. 96. [citado 15 de octubre de 2019]. Disponible en: https://profamilia.org.co/investigaciones/ends/

19. Ministerio de Salud y Protección Social. . Bogotá: MinSalud; 2013. Disponible en: https://www.minsalud.gov.co/Documentos\%20y\%20Publ icaciones/Plan\%20Decenal\%20-

$\% 20$ Documento $\% 20$ en $\% 20$ consulta $\% 20$ para $\% 20$ aprobaci \%C3\%B3n.pdf

20. Ministerio de Salud. Prevalencia del VIH o SIDA entre la población de 15 y 49 años. Dirección de Epidemiología y Demografía y Dirección de Promoción y Prevención. Bogotá, noviembre de 2016. [citado 18 de noviembre de 2019]. Disponible

en: https://www.minsalud.gov.co/sites/rid/Lists/BibliotecaDi gital/RIDE/VS/ED/GCFI/estima-prevalencia-vih-15-492016.pdf

21. Ministerio de Salud y Protección Social. Situación de las infecciones de transmisión sexual diferentes al VIH. Colombia 2009 - 2011 [Internet]. Bogotá: Minsalud; 2013 [citado 23 de febrero de 2018]. Disponible en: www.minsalud.gov.co/salud/Documents/observatorio_vih /documentos/monitoreo_evaluacion/1_vigilancia_salud_pu blica/a_situacion_epidimiologica/SITUACION DE LAS INFECCIONES DE TRANSMISION1.pdf

22. Leftwich HK, Alves MVO. Adolescent Pregnancy. Pediatr Clin North Am. abril de 2017;64(2):381-8. https://doi.org/10.1016/j.pcl.2016.11.007

23. Walsh S, Bruckauf Z, Gaspar T. Adolescents at Risk Psychosomatic health complaints, low life satisfaction, excessive sugar consumption and their relationship with cumulative risks [Internet]. Florence: UNICEF; 2016 [citado 23 de febrero de 2018]. Disponible en: https://www.unicefirc.org/publications/844-adolescents-at-riskpsychosomatic-health-complaints-low-life-satisfactionexcessive.html

24. Ministerio de Salud y Protección Social, Instituto Nacional de Salud, Bienestar Familiar, Universidad Nacional de Colombia. Encuesta Nacional de la situación Nutricional ENSIN 2015. [Internet]. Bogotá: MinSalud; 2017 [citado 23 de febrero de 2018]. Disponible en: https://www.minsalud.gov.co/sites/rid/Lists/BibliotecaDi gital/RIDE/VS/ED/PSP/presentacion-lanzamiento-ensin2015.pdf

25. Hernández AA, Mancilla-DIaz JM, Rayón GLÁ, Luyando MO, López ML, Guerrero JIM. Edad, Consciencia e Interiorización del Ideal Corporal como Predictores de Insatisfacción y Conductas Alimentarias Anómalas. Rev Colomb Psicol. 2013;22(1):121-33. https://doi.org/10.15446/rcp

26. Jaimes GR, Martínez LD, Barajas DO, Plata CP, Martínez JR, Afanador LC. Validación del cuestionario SCOFF para el cribado de los trastornos del comportamiento alimentario en adolescentes escolarizadas. Aten Primaria. 2005;35(2):89-94. https://doi.org/10.1157/13071915

27. Zeiler M, Waldherr K, Philipp J, Nitsch M, Dür W, Karwautz A, et al. Prevalence of Eating Disorder Risk and Associations with Health-related Quality of Life: Results from a Large School-based Population Screening: Prevalence of Eating Disorder Risk. Eur Eat Disord Rev. 2016;24(1):9-18. https://doi.org/10.1002/erv.2368

28. Organización Panamericana de la Salud. Encuesta Mundial de Salud a Escolares (GSHS) Antecedentes, Propósito y Metodología [Internet]. Washington: OPS; 2012 [citado 6 de junio de 2019]. Disponible en: https://www.paho.org/hq/dmdocuments/2016/2012GSHS-Background-Methodology-Sp.pdf

29. Organización Panamericana de la Salud. Encuesta Mundial de Salud Escolar (GSHS) Módulos del cuestionario básico [Internet]. Washington: OPS; 2012 [citado 6 de junio de 2019]. Disponible en: https://www.paho.org/hq/index.php?lang=es

30. Organización Panamericana de la Salud, CDC, OMS, UNICEF, UNESCO. Global School-based Student Health Survey GSHS [Internet]. Washington: OPS; 2012. Disponible en: https://www.paho.org/hq/index.php?option=com_docman \&view=download\&category_slug=gshs-9349\&alias=44268gshs-overview-pdf-268\&Itemid=270\&lang=es

31. Ministerio de Salud. Panorama de la salud de los escolares en Colombia [Internet]. Bogotá: MinSalud; 2018 [citado 6 de junio de 2019]. Disponible en: https://www.minsalud.gov.co/Paginas/Panorama-de-lasalud-de-los-escolares-en-Colombia.aspx

32. Centers for Disease Control and Prevention. Youth Risk Behavior Surveillance System (YRBSS) Overview [Internet]. What is the Youth Risk Behavior Surveillance System (YRBSS)?. USA: CDC; 2018 [citado 6 de junio de 2019]. Disponible

en: https://www.cdc.gov/healthyyouth/data/yrbs/overview.h tm

33. Gadomski A, Bennett S, Young M, Wissow L. Guidelines for Adolescent Preventive Services: the GAPS in practice. Arch Pediatr Adolesc Med. 2003;157:426-32. https://doi.org/10.1001/archpedi.157.5.426

34. Salerno J, Marshall VD, Picken EB. Validity and Reliability of the Rapid Assessment for Adolescent Preventive Services Adolescent Health Risk Assessment. J Adolesc Health. 2012;50(6):595-9. https://doi.org/10.1016/j.jadohealth.2011.10.015

35. Suárez TA, Blanco A, Díaz LA. Validación de la versión en español del Rapid Assessment for Adolescent Preventive Services en adolescentes colombianos. Arch Argent Pediatr. 2016;114(5):448-57. https://doi.org/10.5546/aap.2016.448

36. American Academy of Pediatrics. Bright futures guidelines for health supervision of infants, children, and adolescents pocket guide [Internet]. 4th edition. Elk Grove Village, IL: American Academy of Pediatrics; 2017 [citado 23 de febrero de 2018]. Disponible en: brightfutures.aap.org/Bright\%20Futures\%20Documents / BF4_Introduction.pdf

37. Huitrón-Bravo G, Denova-Gutiérrez E, Halley-Castillo E, Santander-Rigollet S, Bórquez-Puga M, Zapata-Pérez L, et al. Conductas de riesgo en una muestra de adolescentes chilenos y mexicanos: un estudio comparativo. Papeles Poblac. 2011;17(70):33-47. Disponible en: https://www.redalyc.org/articulo.oa?id=11221584003 
38. Departamento Administrativo Nacional de Estadística. Encuesta de Comportamientos y Factores de Riesgo en Niñas, Niños y Adolescentes Escolarizados 2016. Bogotá: DANE; 2017. Disponible en: https://www.dane.gov.co/files/investigaciones/boletines/ ecas/bol_ecas_2016.pdf

39. Breivik K, Olweus D. An item response theory analysis of the Olweus Bullying scale: Olweus Bullying Scale. Aggress Behav. enero de 2015;41(1):1-13. https://doi.org/10.1002/ab.21571

40. Strout TD, Vessey JA, DiFazio RL, Ludlow LH. The Child Adolescent Bullying Scale (CABS): Psychometric evaluation of a new measure. Res Nurs Health. 2018;41(3):252-64. https://doi.org/10.1002/nur.21871

41. Ucañán Robles JD. Propiedades psicometricas del autotest cisneros de acoso escolar en adolescentes del valle Chicama. Rev JANG. 2015;3(1):1-27. Disponible en: http://revistas.ucv.edu.pe/index.php/JANG/article/view/8 $7 / 35$

42. Higuita-Gutiérrez LF, Cardona-Arias JA. Validación de una escala de bullying en adolescentes de instituciones educativas de Medellín, Colombia. Educ Educ. 2017;20(1):724. https://doi.org/10.5294/edu.2017.20.1.1

43. Ronzón-Tirado RC, Muñoz-Rivas MJ, Zamarrón Cassinello MD, Redondo Rodríguez N. Cultural Adaptation of the Modified Version of the Conflicts Tactics Scale (M-CTS) in Mexican Adolescents. Front Psychol. 2019;10:1664-78. https://doi.org/10.3389/fpsyg.2019.00619

44. Rojas NL, Sherrit L, Harris S, Knight JR. The Role of Parental Consent in Adolescent Substance Use Research. J Adolesc Health. https://doi.org/10.1016/j.jadohealth.2007.07.011

45. Cote-Menendez M, Uribe-Isaza MM, Prieto-Suárez E. Validación para Colombia de la escala CRAFFT para tamización de consumo de sustancias psicoactivas en adolescentes. Rev Salud Pública. 2013;15:220-32. https://doi.org/10.15446/rsap

46. Campo-Arias A, Díaz-Martínez LA, Rueda-Jaimes GE, Martínez-Mantilla JA, Amaya-Naranjo W, Campillo HA. Consistencia interna y análisis factorial del cuestionario SCOFF para tamizaje de trastorno de conducta alimentaria en adolescentes estudiantes: Una comparación por género. Univ Psychol. 2006;5(2):295-304. Disponible en: http://www.scielo.org.co/scielo.php?script=sci_arttext\&pi $\mathrm{d}=\mathrm{S} 1657-92672006000200008$

47. Herrán OF, Ardila MF. Internal validity and reliability of the CAGE test in Bucaramanga, Colombia. Biomédica. 2005;25(2):231-41. Disponible en: http://www.scielo.org.co/scielo.php?script=sci_arttext\&pi $\mathrm{d}=\mathrm{S} 0120-41572005000200010$

48. Hagel LD, Mainieri AS, Zeni CP, Wagner MB. Brief report: Accuracy of a 16-item questionnaire based on the HEADSS approach (QBH-16) in the screening of mental disorders in adolescents with behavioral problems in secondary care. J Adolesc. 2009;32(3):753-61. https://doi.org/10.1016/j.adolescence.2008.08.009

49. Spears GV, Stein JA, Koniak-Griffin D. Latent growth trajectories of substance use among pregnant and parenting adolescents. Psychol Addict Behav. 2010;24(2):322-32. https://doi.org/10.1037/a0018518

50. Ministerio de Salud y Protección Social. Desarrollos técnicos, instrumentos y herramientas para la atención en salud sexual y reproductiva y salud mental en contextos de emergencias humanitarias. Bogotá: MinSalud; 2012.
Disponible

en:

https://www.minsalud.gov.co/sites/rid/Lists/BibliotecaDi gital/RIDE/VS/PP/ENT/Desarrollos-tecnicos-

instrumentos-herramientas-1.pdf

51. Ministerio de Salud y Protección Social. Resolución 3280 de 2018. Bogotá: MinSalud; 2018. Disponible en: https://www.minsalud.gov.co/Normatividad_Nuevo/Resol uci\%C3\%B3n\%20No.\%203280\%20de\%2020183280.pdf

52. Ospina F, Hinestrosa MF, Paredes MC. Síntomas de ansiedad y depresión en adolescentes escolarizados de 10 a 17 años en Chía, Colombia. Rev Salud Pública. 2011;13(6):908-20. Disponible

en: https://www.scielosp.org/article/rsap/2011.v13n6/908920/

53. Guerra C, del Río FJ, Morales IM, Cabello F. Validación de la versión reducida para adolescentes de la Encuesta revisada de opinión sexual. Rev Int Andrología. 2017;15(4):135-40. https://doi.org/10.1016/j.androl.2016.10.006

54. Bello Villanueva AM, Palacio J, Rodríguez Díaz M. Medición de la Intención en la Actividad Sexual en Adolescentes: una Aproximación de Acuerdo al Género del Caribe Colombiano. Ter Psicológica. 2013;31(3):343-53. http://dx.doi.org/10.4067/S0718-48082013000300009

55. Morgan JF, Reid F, Lacey JH. The SCOFF questionnaire: assessment of a new screening tool for eating disorders. Bmj. 1999;319(7223):1467-8. DOI: 10.1136/bmj.319.7223.1467

56. Rueda GE, Díaz LA, Campo A, Barros JA, Osorio BC, del Pilar L. Validación de la encuesta SCOFF para tamizaje de trastornos de la conducta alimentaria en mujeres universitarias. Biomédica. 2005;25(2):196-202. https://doi.org/10.7705/biomedica.v25i2.1342

57. Manrique F, Barrera L, Ospina JM. Prevalencia de bulimia y anorexia en adolescentes de Tunja y algunos factores de riesgo probablemente asociados. Av En Enferm. 2006;XXIV(2):38-46. Disponible en https://revistas.unal.edu.co/index.php/avenferm/article/v iew/35930/37039

58. Brandt LMT, Fernandes LHF, Aragão AS, Luna TP da C, Feliciano RM, Auad SM, et al. Risk behavior for bulimia among adolescents. Rev Paul Pediatr. 2019;37(2):217-24. https://doi.org/10.1590/1984-0462/;2019;37;2;00008

59. Unikel C, Bojórquez L, Carreño S. Validación de un cuestionario breve para medir conductas alimentarias de riesgo. Salud Pública México. 2004;46(6):509-15. Disponible http://www.scielo.br/pdf/spm/v46n6/22563.pdf

60. Pichot P. DSM-IV: manual diagnóstico y estadistico de los trastornos mentales. Barcelona: Masson SA; 1995. Disponible en: http://www.mdp.edu.ar/psicologia/psico/cendoc/archivo s/Dsm-IV.Castellano.1995.pdf

61. Haugland S, Wold B. Subjective health complaints in adolescence-Reliability and validity of survey methods. J Adolesc. octubre de 2001;24(5):611-24. https://doi.org/10.1006/jado.2000.0393

62. Currie C, Griebler R, Inchley J, Theunissen A, Molcho M, Samdal O, Dür W. Health behaviour in school-aged children (HBSC) Study protocol: Background, Methodology and Mandatory items for the 2009/10 Survey [Internet]. Edinburgh: CAHRU \& Vienna: LBIHPR; 2010. Disponible en: https://www.uib.no/sites/w3.uib.no/files/attachments/h bsc_external_study_protocol_2009-10.pdf 
63. Pérez C, Aranceta J, Salvador G, Varela G. Food Frequency Questionnaires. Nutr Hosp. 2015;3(3):49-56. DOI: 10.3305/nh.2015.31.sup3.8751

64. Webb MJ, Kauer SD, Ozer EM, Haller DM, Sanci LA. Does screening for and intervening with multiple health compromising behaviours and mental health disorders amongst young people attending primary care improve health outcomes? A systematic review. BMC Fam Pract. 2016;17:104. https://doi.org/10.1186/s12875-016-05041
65. Foti K, Balaji A, Shanklin S. Uses of Youth Risk Behavior Survey and School Health Profiles data: applications for improving adolescent and school health. J Sch Health. 2011;81(6):345-54. 1561.2011 .00601 https://doi.org/10.1111/j.1746- 